\title{
Higher Body Mass Index Is an Independent Predictor of Left Atrial Enlargement
}

\author{
Prasanna Venkatesh Kumar, Aman Mundi, Gloria Caldito, Pratap C. Reddy \\ Division of Cardiology, Department of Medicine, Louisiana State University Health Sciences Center, Shreveport, USA. \\ Email: pkumar@prairieheart.com
}

Received August 25 ${ }^{\text {th }}, 2011$; revised October $8^{\text {th }}, 2011$; accepted October $20^{\text {th }}, 2011$.

\begin{abstract}
Background: The influence of obesity on the cardiac geometry is less clear. Our objective was to determine if body mass index (BMI) was an independent predictor of left atrial enlargement (LAE) relative to LV thickness, dimension and function. Methods: Retrospective analysis of 90 consecutive patients with available echocardiographic data was performed. Patients were categorized into those with LAE (defined as $>4.0 \mathrm{~cm}$ in transverse dimension by $M$-mode, $n=$ $36)$ and those with normal LA size $(n=54)$. The clinical and echocardiographic variables were compared between the two groups. Results: Compared to patients with normal LA size, patients with LAE had significantly higher BMI (32 \pm 8 vs $27 \pm 8, p=0.006$ ), greater LV thickness, increased LV cavity dimension, and depressed LV systolic function. BMI was a strong and independent predictor of LAE. Left ventricular thickness and LV end-diastolic (LVED) dimension were other independent predictors of LAE. After adjusting for the significant effects of LV thickness and LV diameter, every unit increase in BMI resulted in an 8\% increase in the odds of having LAE. Conclusions: 1) Higher BMI is an independent predictor of $L A E$. 2) Presence of $L V$ enlargement and $L V$ systolic dysfunction in these patients suggests that in the face of higher BMI, cardiac workload may exceed the compensatory LV hypertrophy and 3) LAE may be a consequence of the greater force developed by the LA to fill the LV.
\end{abstract}

Keywords: Body Mass Index, Left Atrial Size, Obesity, Cardiomyopathy

\section{Introduction}

Obesity has been shown to influence cardiac mass [1]. Subclinical left ventricular (LV) diastolic dysfunction is present in all grades of isolated obesity and correlates with BMI [2]. Overweight and obesity are associated with LV hypertrophy (LVH) and contractile impairment in patients with underlying chronic LV volume overload [3]. In patients with uncontrolled hypertension, obesity has been associated with increased left atrial (LA) size [4]. However it is less clear if higher BMI is an independent predictor of LA enlargement (LAE).

There are several determinants of LA size. These include pressure and volume overload, diastolic dysfunction and body size (body surface area) [5]. However, the effect of BMI on cardiac geometry is less clear. The purpose of this study was to evaluate the effect of BMI on left atrial size. Since LAE is hypothesized to be the end result of the effects of obesity on LV geometry, we also compared LV thickness, dimensions and function in patients with and without LAE.

\section{Methods}

\subsection{Study Population}

Ninety consecutive patients from a major university teaching hospital with available echocardiographic data were included in this retrospective analysis. Patients were categorized into those with LAE $(n=36)$ and those with normal LA size $(n=54)$.

\subsection{Definitions}

Obesity was defined as BMI $\geq 30$. LAE was defined as LA size $>4.0 \mathrm{~cm}$ in transverse dimension measured by M-mode. M-mode and $2 \mathrm{D}$ measurements of LV size and function, derived from parasternal long axis measures of LV end diastolic (LVED) and LV end systolic (LVES) dimensions were compared between the two groups. Left ventricular enlargement was defined as LVED $\geq 56 \mathrm{~mm}$ and LVES $\geq 40 \mathrm{~mm}$. Standard echocardiographic criteria were used to determine systolic and diastolic dysfunction. 


\section{Results}

The clinical and echocardiographic characteristics are depicted in Table 1. Patients with LAE had significantly higher BMI ( $32 \pm 8$ vs. $27 \pm 8, \mathrm{p}=0.006)$ compared to those without LAE. Patients with LAE also had significantly increased LV thickness, increased LVED and LVES dimension, and lower LV ejection fraction (LVEF) compared to those with normal LA size (Table 1).

\section{Statistical Analysis}

Data was analyzed to determine the independent signifycant predictors of LAE. A univariate logistic regression analyses (Table 2) shows the factors observed to be significantly associated with LAE. With the exception of
LVEF which was inversely associated with LAE, BMI, interventricular septal thickness (IVST), Posterior LV wall thickness (PLVW), LVES and LVED were all directly and significantly associated with LAE. The independent significant predictors for LAE were BMI, PLVW and LVED (Table 3). The adjusted OR for each independent significant predictor was the OR for the factor adjusted for the effect of the two other independent significant factors. Every unit increase in BMI resulted in an $8 \%$ increase in the odds for a patient to develop LAE. For every unit increase in PLVW and LVED, the odds of a patient developing LAE increased $31 \%$ and $9 \%$ respectively, when adjusted for the significant effects of the other two predictors.

Table 1. Clinical and echocardiographic variables in patients with and without left atrial enlargement (LAE).

\begin{tabular}{|c|c|c|c|}
\hline Clinical variable & $\begin{array}{c}\text { Patients with LAE } \\
\mathrm{N}=36\end{array}$ & $\begin{array}{l}\text { Patients with normal LA size, } \\
\qquad N=54\end{array}$ & p-value \\
\hline Mean age \pm SD (years) & $52 \pm 12$ & $50 \pm 14$ & ns \\
\hline Percentage of males & $20 / 36(55.5 \%)$ & $21 / 54(39 \%)$ & $\mathrm{ns}$ \\
\hline Left atrial size (mm) & $47.25 \pm 6.25$ & $33.46 \pm 4.82$ & $<0.001 *$ \\
\hline BMI & $32 \pm 8$ & $27 \pm 8$ & $0.006^{*}$ \\
\hline Diabetes & $36 \%$ & $30 \%$ & ns \\
\hline Hypertension & $81 \%$ & $76 \%$ & $\mathrm{~ns}$ \\
\hline Systolic Blood Pressure & $140 \pm 20$ & $135 \pm 27$ & ns \\
\hline Diastolic Blood Pressure & $80 \pm 12$ & $76 \pm 17$ & ns \\
\hline Coronary Artery Disease & $10 / 36(27 \%)$ & $9 / 54(17 \%)$ & $\mathrm{ns}$ \\
\hline Atrial fibrillation & $4 / 36(11 \%)$ & $2 / 54(4 \%)$ & ns \\
\hline LV ejection fraction (\%) & $45 \pm 22$ & $55 \pm 14$ & $0.007 *$ \\
\hline Posterior LV wall thickness (mm) & $12.42 \pm 3.08$ & $10.75 \pm 2.09$ & $0.003^{*}$ \\
\hline Interventricular septal thickness (mm) & $12.76 \pm 3.69$ & $10.65 \pm 2.14$ & $0.001 *$ \\
\hline LV End Diastolic Diameter (mm) & $55.09 \pm 12.16$ & $49.44 \pm 6.75$ & $0.006^{*}$ \\
\hline LV End Systolic Diameter (mm) & $40.23 \pm 14.53$ & $32.67 \pm 8.05$ & $0.002 *$ \\
\hline Diastolic dysfunction & $15 / 36(42 \%)$ & $17 / 54(32 \%)$ & $\mathrm{ns}$ \\
\hline Mitral Regurgitation & $10 / 36(28 \%)$ & $6 / 54(11 \%)$ & $\mathrm{ns}$ \\
\hline Mitral Stenosis & none & none & $\mathrm{ns}$ \\
\hline
\end{tabular}

${ }^{*} \mathrm{p}<0.05$ is significant; $\mathrm{ns} —$ not significant. SD—standard deviation; BMI—body mass index, $\mathrm{LV} —$ left ventricle. 
Table 2. Factors significantly associated with LAE using separate simple logistic regression analysis.

\begin{tabular}{cccc}
\hline Factor & $\begin{array}{c}\text { Odds Ratio } \\
(\text { OR })\end{array}$ & $\begin{array}{c}95 \% \text { CI for } \\
\text { OR }\end{array}$ & p-value \\
\hline BMI & 1.08 & 1.02 to 1.14 & $0.008^{* *}$ \\
IVST & 1.30 & 1.09 to 1.55 & $0.004^{* *}$ \\
LVES & 1.06 & 1.02 to 1.11 & $0.006^{* *}$ \\
LVED & 1.07 & 1.02 to 1.13 & $0.012^{*}$ \\
LVEF & 0.97 & 0.94 to 0.99 & $0.012^{*}$ \\
PLVW & 1.30 & 1.07 to 1.58 & $0.008^{* *}$ \\
\hline
\end{tabular}

*Significant at $5 \%$ level $(0.01<$ p-value $<0.05) * *$ Significant at $1 \%$ level (p-value < 0.01) BMI-body mass index; IVST - Interventricular septal thickness, LVES — Left ventricular end systolic diameter; LVED_Left ventricular end-diastolic diameter; LVEF-Left ventricular ejection fraction; PLVW-Posterior left ventricular wall thickness.

Table 3. Independent significant predictors of LAE.

\begin{tabular}{cccc}
\hline Predictor & Adjusted OR & $95 \%$ CI for OR & p-value \\
\hline BMI & 1.08 & 1.01 to 1.15 & $0.02^{*}$ \\
PLVW & 1.31 & 1.14 to 1.73 & $0.002^{* *}$ \\
LVED & 1.09 & 1.03 to 1.16 & $0.006^{* *}$ \\
\hline
\end{tabular}

BM-body mass index; PLVW-Posterior left ventricular wall thickness; LVED — Left ventricular end diastolic diameter; OR — odds ratio; *p value is significant

\section{Discussion}

Obesity is a known determinant of cardiovascular risk. The Framingham Heart Study showed that overweight and obese patients have an increased risk of hypertension and cardiovascular sequelae [6]. These include coronary heart disease, atrial fibrillation and stroke [6]. Obesity is also associated with an increased risk of heart failure, proportionate to the degree of increase in BMI [7]. The term Obesity Cardiomyopathy is applied when obesity induced cardiac structural and hemodynamic changes result in congestive heart failure. Obesity Cardiomyopathy typically occurs in persons with severe and long standing obesity $[8,9]$. Bariatric surgery has been shown to improve cardiac function in morbidly obese patients with severe Cardiomyopathy [10]. These observations make it important to study the structural, geometric and functional effects of obesity on the heart to better understand the pathophysiology of Obesity Cardiomyopathy.
In our study, BMI was significantly higher in patients with LAE compared to those with normal left atrial size. We also found BMI to be an independent predictor of LAE. After adjusting for the significant effects of LVH and LV diameter, every unit of increase in BMI resulted in an $8 \%$ increase in the odds of developing LAE. Our findings of the influence of BMI on LA size are supported by a recent prospective population study which showed that both obesity and hypertension were independent predictors of LAE (odds ratio for obesity was $2.4, \mathrm{p}<0.001$, which was greater than that for hypertension which had odds ratio of 2.2, $\mathrm{p}<0.001$ ) [11].

Among the six factors that were significantly associated with LAE only BMI, PLVW and LVED were independent significant predictors of LAE. Patients with LAE were more likely to be obese, have increased LV wall thickness, larger LV dimension and reduced LV systolic function compared to those with normal LA size.

The proposed mechanisms for LAE in obese patients are illustrated in Figure 1. Obesity causes an increase in total blood volume and cardiac output and is associated with elevated cardiac filling pressures. This hypercirculation leads to LV dilatation or can cause compensatory $\mathrm{LVH}$ and diastolic dysfunction. The LV dilatation can lead to increased wall stress which can lead to secondary or eccentric hypertrophy of the left ventricle. Presence of chronically elevated wall stress can lead to LV systolic dysfunction which in the presence of increased cardiac work load (exceeding the compensatory hypertrophy) can result in LAE. The strong association of LV wall thickness, LV chamber size and LV systolic function with LAE in our study suggests that LAE likely resulted from the effects of obesity on LV geometry.

Obesity has been well recognized to be a risk factor for atrial fibrillation [12]. In obese patients, atrial fibrillation likely results from multiple factors, LAE being one of them. In the present study, although the prevalence of atrial fibrillation was greater in the group with LAE compared to those without ( $11 \%$ vs. $4 \%)$ the difference was not statistically significant possibly due to the small number of study patients. In the population based study by Pritchett et al., left atrial volume adjusted to body surface area was more associated with cardiovascular diseases than the dimension by M-mode measurement [13]. Our results suggest that higher BMI is an independent predictor of left atrial enlargement using left atrial dimension measurements.

\section{Study Limitations}

The retrospective design and the small number of patients are potential limitations of this study. The non-randomized nature of the study and the non-homogeneity of the population leave the possibility of selection bias. 


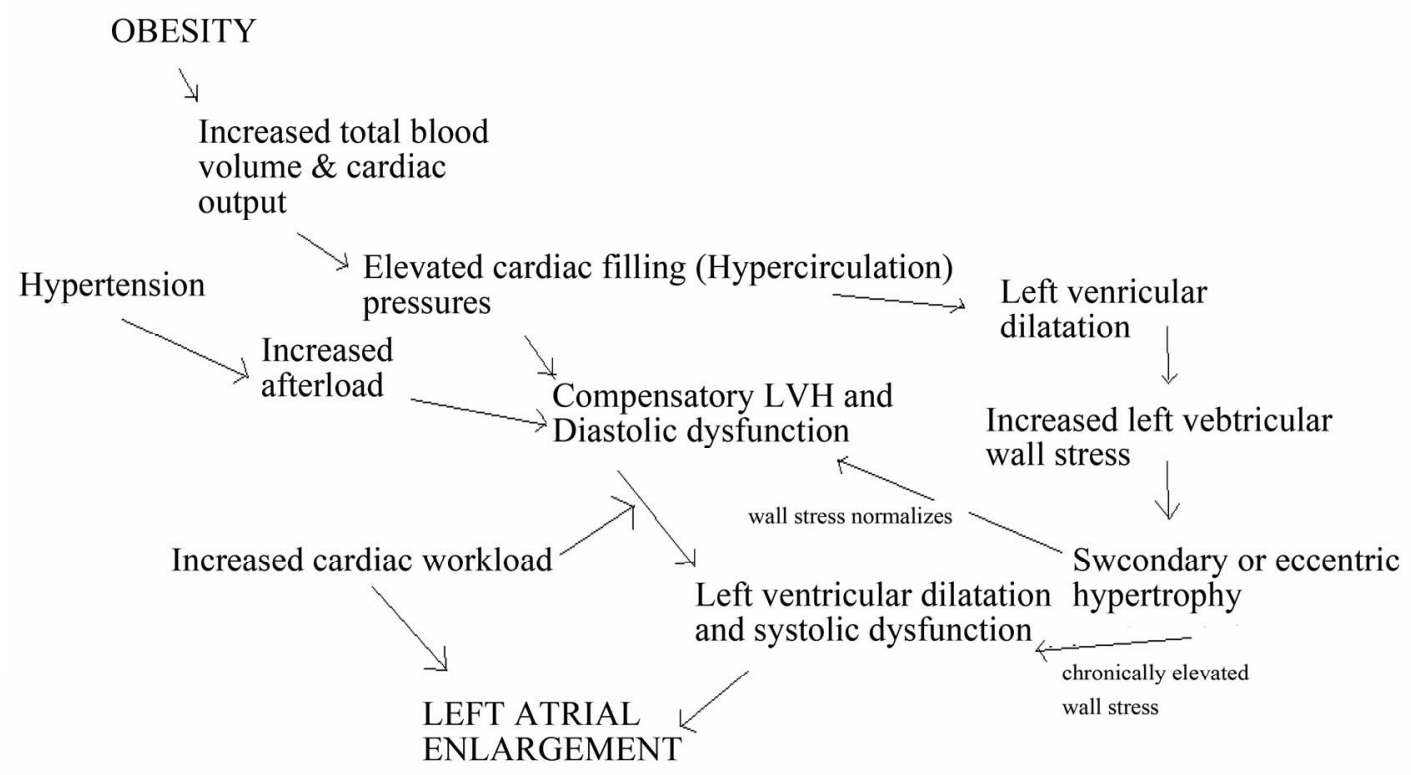

Figure 1. Proposed mechanisms for obesity induced left atrial enlargement.

\section{Conclusions}

Higher BMI is an independent predictor of LAE and likely the result of its influence on cardiac geometry. In addition to $\mathrm{LVH}$, the presence of $\mathrm{LV}$ enlargement and LV systolic dysfunction in patients with LAE suggests that in the face of higher BMI, cardiac workload may exceed the compensatory LV hypertrophy. LAE may be a consequence of the greater force developed by the left atrium to fill the LV. The effect of obesity on left atrial size provides one explanation for the higher incidence of atrial fibrillation in obese patients.

\section{REFERENCES}

[1] C. Y. Wong, T. O’Moore-Sullivan, R. Leano, N. Byrne, E. Beller and T. H. Marwick, "Alterations of Left Ventricular Myocardial Characteristics Associated with Obesity," Circulation, Vol. 110, No. 19, 2004, pp. 3081-3087. doi:10.1161/01.CIR.0000147184.13872.0F

[2] M. Pascual, D. A. Pascual, F. Soria, T. Vicente, A. M. Hernandez, F. J. Tebar and M. Valdes, "Effects of Isolated Obesity on Systolic and Diastolic Left Ventricular Function," Heart, Vol. 89, 2003, pp. 1152-1156. doi:10.1136/heart.89.10.1152

[3] P. Ballo, A. Motto, S. Mondillo and S. A. Faraguti, "Impact of Obesity on Left Ventricular Mass and Function in Subjects with Chronic Volume Overload," Obesity, Vol. 15, No. 8, 2007, pp. 2019-2026. doi:10.1038/oby.2007.241

[4] J. S. Gottdiener, D. J. Reda, D. W. Williams and B. J. Materson, "Left Atrial Size in Hypertensive Men: Influence of Obesity, Race and Age," Journal of the American College of Cardiology, Vol. 29, No. 3, 1997, pp. 651-658.

\section{doi:10.1016/S0735-1097(96)00554-2}

[5] W. P. Abhayaratna, J. B. Seward, C. P. Appleton, P. S. Douglas, J. K. Oh, A. J. Tajik and T. S. Tsang, "Left Atrial Size. Physiologic Determinants and Clinical Applications," Journal of the American College of Cardiology, Vol. 47, No. 12, 2006, pp. 2357-2363. doi:10.1016/i.jacc.2006.02.048

[6] P. W. Wilson, R. B. D’Agostino, L. Sullivan, H. Parise and W. B. Kannel, "Overweight and Obesity as Determinants of Cardiovas Cular Risk: The Framingham Experience," Archives of Internal Medicine, Vol. 162, No. 16, 2002, pp. 1867-1872. doi:10.1001/archinte.162.16.1867

[7] S. Kenchaiah, J. C. Evans, D. Levy, P. W. Wilson, Ben E. J. Jamin, M. G. Larson, W. B. Kannel and R. S. Vasan, "Obesity and the Risk of Heart Failure," New England Journal of Medicine, Vol. 347, No. 5, 2002, pp. 305-313. doi:10.1056/NEJMoa020245

[8] J. K. Alexander and M. A. Alpert, "Pathogenesis and Clinical Mani Festations of Obesity Cardiomyopathy," In: M. A. Alpert and J. K. Alexander, Eds., The Heart and Lung in Obesity, Futura, Armonk, 1998, pp. 133-146.

[9] J. M. McGavock, I. Lingvay, I. Zib, T. Tillery, N. Salas, R. Unger, B. D. Levine, P. Raskin, R. G. Victor and L. S. Szczepaniak, "Cardiac Steatosis in Diabetes Mellitus: A ${ }^{1}$ H-Magnetic Resonance Spectroscopy Study," Circulation, Vol. 116, No. 10, 2007, pp. 1170-1175. doi:10.1161/CIRCULATIONAHA.106.645614

[10] C. A. McCloskey, G. V. Ramani, M. A. Mathier, P. R. Schauer, G. M. Eid, S. G. Mattar, A. P. Courcoulas and R. Ramanathan, "Bari Atric Surgery Improves Cardiac Function in Morbidly Obese Patients with Severe Cardiomyopathy," Surgery for Obesity and Related Diseases, Vol. 3, No. 5, 2007, pp. 503-507. doi:10.1016/j.soard.2007.05.006 
[11] J. Stritzke, M. R. P. Markus, S. Duderstadt, W. Lieb, A. Luchner, A. Döring, U. Keil, H. W. Hense and Schunkert Hand MONICA/KORA Investigators, "The Aging Process of the Heart: Obesity Is the Main Risk Factor for Left Atrial Enlargement during Aging: The MONICA/KORA (Monitoring of Trends and Determinations in Cardiovascular Disease/Cooperative Research in the Region of Augsburg) Study," Journal of the American College of Cardiology, Vol. 54, No. 21, 2009, pp. 1982-1989. doi:10.1016/j.jacc.2009.07.034

[12] T. J. Wang, H. Parise, D. Levy, R. B. Sr. D’Agostino, P.
A. Wolf, R. S. Vasan and E. J. Benjamin, "Obesity and the Risk of New-Onset Atrial Fibrillation," Framingham Heart Study, Japan Automobile Manufacturers Association, Vol. 292, No. 20, 2004, pp. 2471- 2477.

[13] A. M. Pritchett, S. J. Jacobsen, D. W. Mahoney, R. J. Rodeheffer, K. R. Bailey and M. M. Redfield, "Left Atrial Volume as an Index of Left Atrial Size: A Population-Based Study," Journal of the American College of Cardiology, Vol. 41, No. 6, 2003, pp. 1036-1043. doi:10.1016/S0735-1097(02)02981-9 